\title{
Conflict-Free Colouring of Graphs
}

\section{Journal Article}

\section{Author(s):}

Glebov, R.; Szabo, T.; Tardos, G.

Publication date:

2014-05

\section{Permanent link:}

https://doi.org/10.3929/ethz-b-000087236

\section{Rights / license:}

In Copyright - Non-Commercial Use Permitted

Originally published in:

Combinatorics, Probability \& Computing 23(3), https://doi.org/10.1017/S0963548313000540 


\title{
Conflict-Free Colouring of Graphs
}

\author{
ROMAN GLEBOV ${ }^{1 \dagger}$, TIBOR SZABÓ ${ }^{2}$ and GÁBOR TARDOS \\ ${ }^{1}$ Department of Mathematics, ETH, 8092 Zurich, Switzerland \\ and \\ Mathematics Institute and DIMAP, University of Warwick, Coventry CV4 7AL, UK \\ (e-mail: roman.1.glebov@gmail.com) \\ ${ }^{2}$ Institute of Mathematics, Free University of Berlin, 14195 Berlin, Germany \\ (e-mail: szabo@math.fu-berlin.de)
}

${ }^{3}$ Alfréd Rényi Institute of Mathematics, Hungarian Academy of Sciences, Budapest, Hungary and

Zhejiang Normal University, Jinhua, China

(e-mail: tardos@renyi.hu)

Received 23 November 2011; revised 20 September 2013; first published online 29 November 2013

\begin{abstract}
We study the conflict-free chromatic number $\chi_{C F}$ of graphs from extremal and probabilistic points of view. We resolve a question of Pach and Tardos about the maximum conflict-free chromatic number an $n$-vertex graph can have. Our construction is randomized. In relation to this we study the evolution of the conflict-free chromatic number of the Erdoss-Rényi random graph $G(n, p)$ and give the asymptotics for $p=\omega(1 / n)$. We also show that for $p \geqslant 1 / 2$ the conflict-free chromatic number differs from the domination number by at most 3 .
\end{abstract}

2010 Mathematics subject classification: Primary 05C15

Secondary 05C35, 05C80, 05D40, 05C69

\section{Introduction and definitions}

Let $G=(V, E)$ be a simple graph. For every $x \in V$ we denote by $N(x)=\{y \in V: x y \in E\}$ its neighbourhood and by $N[x]=N(x) \cup\{x\}$ its closed neighbourhood. A (not necessarily proper) vertex colouring $\chi$ of $G$ is called conflict-free if, for each vertex $x \in V$, there exists

$\dagger$ This research was done when the author was affiliated with the Institute of Mathematics, Free University of Berlin. The author was supported by DFG within the research training group 'Methods for Discrete Structures'.

‡ Partially supported by an NSERC grant, the MTA Cryptography 'Lendület' project, and the Hungarian OTKA grant NN-102029. 
a vertex $y$ in $N[x]$ whose colour is different from the colour of each other vertex in $N[x]$. We then say that $y$ has a unique colour in $N[x]$. The conflict-free chromatic number $\chi_{C F}(G)$ is the smallest $r$ such that there exists a conflict-free $r$-colouring of $G$. Conflict-free colouring can be interpreted as a relaxation of the usual proper colouring concept where each vertex $x$ is required to have a unique colour in its own closed neighbourhood $N[x]$. Hence $\chi_{C F}(G) \leqslant \chi(G)$ for every graph $G$.

The study of conflict-free colourings originated in the work of Even, Lotker, Ron and Smorodinsky [4] and Smorodinsky [9], who were motivated by the problem of frequency assignment in cellular networks. (See the recent survey by Smorodinsky [10].) In most of these classical instances the graphs studied arise from a geometric setting. Recently Pach and Tardos [7] initiated the study of the problem for abstract graphs and hypergraphs. Here we continue the consideration of conflict-free colourings of abstract graphs.

Note that, unlike the proper colouring number, the conflict-free chromatic number is not monotone. In particular, in the two extremes, $\chi_{C F}\left(K_{n}\right)=2$ for the complete graph and $\chi_{C F}\left(\bar{K}_{n}\right)=1$ for the empty graph, while the conflict-free chromatic number of general graphs can be arbitrarily high. We investigate this parameter from extremal and probabilistic points of view.

Pach and Tardos [7] raised the problem of determining the order of magnitude of $\chi_{C F}(n):=\max \left\{\chi_{C F}(G):|V(G)|=n\right\}$, the largest conflict-free chromatic number an $n$ vertex graph can have. From above they showed $\chi_{C F}(n)=O\left(\ln ^{2} n\right)$, but from below they could only prove that the conflict-free colouring number of the random graph $G\left(n, \frac{1}{2}\right)$ is asymptotically almost surely $\Omega(\ln n)$, hence $\chi_{C F}(n)=\Omega(\ln n)$. Here 'asymptotically almost surely' means probabilities tending to 1 as $n$ goes to infinity, and it will be abbreviated below as a.a.s.

First one could try to improve the lower bound $\chi_{C F}(n)$ by considering the random graph $G(n, p)$ with some $p=p(n) \neq 1 / 2$. In our first theorem we give tight estimates (holding a.a.s.) for the conflict-free chromatic number of these random graphs. Our bounds show that some probabilities $p(n) \rightarrow 0$ yield the highest conflict-free colouring numbers for the $G(n, p(n))$, but these are only a constant factor larger than those of $G(n, 1 / 2)$.

To state our theorem we introduce

$$
\mu=\mu(p)=\max \left\{i p(1-p)^{i-1}: i \in \mathbb{N}^{+}\right\}
$$

for $0<p<1$. Note that the maximum is taken at $i=\lfloor 1 / p\rfloor$, so we have

$$
\mu(p)=\left\lfloor\frac{1}{p}\right\rfloor p(1-p)^{\lfloor 1 / p\rfloor-1},
$$

and (as a simple calculation shows) this is a strictly increasing function tending to $e^{-1}$ as $p$ goes to 0 .

Theorem 1.1. For every $\varepsilon>0$ and every function $0<p=p(n)<1-\varepsilon$ such that $n p(n) \rightarrow$ $\infty$, the following holds a.a.s.:

$$
(1-\varepsilon) \frac{\ln (n p)}{-\ln (1-\mu(p))} \leqslant \chi_{C F}(G(n, p)) \leqslant(1+\varepsilon) \frac{\ln (n p)}{-\ln (1-\mu(p))} .
$$


Note that the theorem implies $\chi_{C F}(G(n, p))=O(\log n)$ a.a.s. for all $p$ considered. It is not hard to show that the $O(\log n)$ upper bound is also valid a.a.s. in the full range of $p \in[0,1]$. For the range $p=O(1 / p)$ this follows from $\chi_{C F}(G) \leqslant \chi(G) \leqslant \Delta(G)+1$, but in this range we are not able to determine the asymptotics.

For $1 / 2 \leqslant p<1$ we can prove an even tighter result: the conflict-free colouring number differs by at most 3 from the domination number. A set $S$ of vertices of a graph $G$ constitutes a dominating set if each $v \in V$ is either in $S$ or is adjacent to a vertex in $S$. The domination number $D(G)$ is the smallest size of a dominating set in $G$.

Theorem 1.2. For every graph $G$,

$$
\chi_{C F}(G) \leqslant D(G)+1 .
$$

Furthermore, for $\frac{1}{2} \leqslant p(n)$, a.a.s.

$$
D(G(n, p(n)))-3 \leqslant \chi_{C F}(G(n, p(n))) .
$$

The domination number of the random graph with constant $p$ was pinned down to be one of two integers a.a.s. by Wieland and Godbole [11]. Furthermore, it was observed by Glebov, Liebenau and Szabó [5] that the same result also holds for a variable $p(n)$. The following is a corollary of these results for the range of our interest.

Theorem 1.3 (corollary of $[11,5])$. For $1 / 2 \leqslant p<1$ the domination number $D(G(n, p(n))$ is either

$$
\left\lfloor\frac{\ln n-2 \ln \ln n+\ln \ln \frac{1}{1-p}}{-\ln (1-p)}\right\rfloor+1
$$

or one more a.a.s.

Hence the behaviour of $\chi_{C F}$ is also very well understood in this range. In fact, we prove Theorem 1.2 by calculating the a.a.s. lower bound on $\chi_{C F}(G(n, p))$ and comparing it with the a.a.s. domination number. Note that, using Theorem 1.3, Theorem 1.2 implies Theorem 1.1 for the range $p \geqslant 1 / 2$, where we have $\mu(p)=p$. We mention that for the range $p<\frac{1}{2}$ the results of $[11,5]$ on the domination number and Theorem 1.1 imply that the conflict-free chromatic number and the domination number differ in the asymptotics.

In our final result we resolve the open problem of Pach and Tardos [7] regarding $\chi_{C F}(n)$ by constructing $n$-vertex graphs $G$ with $\chi_{C F}(G)=\Omega\left(\ln ^{2} n\right)$.

\section{Theorem 1.4.}

$$
\chi_{C F}(n)=\Theta\left(\ln ^{2} n\right)
$$

The structure of the paper is as follows: in Section 2 we prove Theorems 1.1 and 1.2, while Theorem 1.4 is proved in Section 3. For simplicity we routinely omit floor and ceiling signs as long as they do not influence the validity of our asymptotic statements. 
Notation. Let $G$ be a graph with vertex set $V=V(G)$ and let $A \subseteq V$. We say that

$$
N_{G}^{(1)}(A)=\{v \in V \backslash A:|N(v) \cap A|=1\}
$$

is the one-neighbourhood of $A$ and

$$
\overline{N_{G}}(A)=V \backslash \bigcup_{x \in A} N[x]
$$

is the non-neighbourhood of $A$. The subscript $G$ is omitted if it is clear from the context.

We use $\left(\begin{array}{l}V \\ m\end{array}\right)$ to denote the set of all $m$-element subsets of $V$.

\section{Evolution of the conflict-free chromatic number in random graphs}

\subsection{Upper bounds}

A simple upper bound is obtained from the fact that any proper colouring is a conflict-free colouring, so

$$
\chi_{C F}(G) \leqslant \chi(G) .
$$

However, this bound is a.a.s. not tight for the random graph $G(n, p)$ in the range of $p$ we are interested in, i.e., for $p=\omega(1 / n)$.

Another inequality involves domination. If a set of vertices $S$ is a dominating set of $G$ then one can construct a conflict-free colouring of $G$ with $|S|+1$ colours by giving $|S|$ distinct colours to the vertices in $S$ and one further colour to vertices in $V(G) \backslash S$. Hence, for every graph $G$,

$$
\chi_{C F}(G) \leqslant D(G)+1 .
$$

This proves the upper bound in Theorem 1.2.

The rest of this section deals with the upper bound in Theorem 1.1.

Regarding conflict-free colourings, the crucial property of a vertex $x$ is whether it has exactly one neighbour in some colour class $S$ and hence the colour of $S$ is unique in $N[x]$. For a fixed set $S$ and a fixed vertex $x \in V \backslash S$ the probability of this happening is $|S| p(1-p)^{|S|-1}$. This motivates our definition of $\mu(p)$ in Section 1 as the maximum of this probability for any colour class size. We let $m=\lfloor 1 / p\rfloor$ stand for the 'most desirable' colour class size maximizing the above probability and giving $\mu=m p(1-p)^{m-1}$.

Since the upper bound in Theorem 1.2 implies the upper bound in Theorem 1.1 for $p \geqslant \frac{1}{2}$, we assume $p<\frac{1}{2}$ from now on. To start with we prove two technical lemmas for random graphs.

First we give an explicit bound on the probability that the domination number of a random graph is extremely low. We need the explicit bound because we will use the union bound for more than a constant number of similar events, and thus the a.a.s. bound of Theorem 1.3 is not enough.

Lemma 2.1. For any $\ell \in \mathbb{N}^{+}$and $p$ with $\frac{100}{\ell}<p<\frac{1}{2}$ we have that

$$
\mathbb{P}[D(G(\ell, p))<m]<0.9^{\ell},
$$

where $m=\lfloor 1 / p\rfloor$ as before. 
Proof. Throughout the proof we will use $m-1<\frac{1}{p}<\frac{\ell}{100}$. Let $S \subset V$ be a set of size $m-1$. The probability that a vertex $x \in V \backslash S$ has no neighbour in $S$ is

$$
\mathbb{P}[N(x) \cap S=\emptyset]=(1-p)^{|S|} \geqslant(1-p)^{1 / p}>1 / 4 .
$$

The events that $N(x) \cap S=\emptyset$ are independent for $x \in V \backslash S$, hence $S$ is dominating with probability $<(3 / 4)^{\ell-m+1}$. The probability in the lemma is

$$
\begin{aligned}
\mathbb{P}\left[\exists S \in\left(\begin{array}{c}
V \\
m-1
\end{array}\right): \bar{N}(S)=\emptyset\right] & <\left(\begin{array}{c}
\ell \\
m-1
\end{array}\right)(3 / 4)^{\ell-m+1} \\
& <0.9^{\ell} .
\end{aligned}
$$

We know that the expected size of the one-neighbourhood of a set of vertices of size $m$ is $(|V|-m) \mu$. The following is a routine observation that the actual size deviates largely from this expectation with a very low probability.

Lemma 2.2. For every $\delta>0$ there exists a $K=K(\delta)$ such that for any $p=p(\ell)>\frac{K}{\ell}$ in $G(\ell, p)$ we have that

$$
\mathbb{P}\left[\exists S \in\left(\begin{array}{l}
V \\
m
\end{array}\right):\left|N^{(1)}(S)\right|<(1-\delta) \mu(\ell-m)\right]<e^{-\frac{\delta^{2}}{4} \mu \ell},
$$

where $m=\lfloor 1 / p\rfloor$ and $\mu=\mu(p)=m p(1-p)^{m-1}$.

Proof. For an arbitrary set $S \subset V$ of size $m$ and vertex $x \in V \backslash S$, the probability that $x$ has exactly one neighbour in $S$ is $\mu$. The random variable $\left|N^{(1)}(S)\right|$ is the sum of $\ell-m$ mutually independent characteristic variables and its expectation is $\mu(\ell-m)$. Hence, by the Chernoff bound and the union bound we have

$$
\begin{aligned}
\mathbb{P}\left[\exists S \in\left(\begin{array}{c}
V \\
m
\end{array}\right):\left|N^{(1)}(S)\right|<(1-\delta) \mu(\ell-m)\right] & <\left(\begin{array}{c}
\ell \\
m
\end{array}\right) e^{-\frac{\delta^{2}}{2} \mu(\ell-m)} \\
& \leqslant\left((e K)^{\frac{1}{K}} e^{-\frac{\delta^{2}}{3} \mu}\right)^{\ell},
\end{aligned}
$$

and the bound follows if $K$ is sufficiently large.

Let us choose $\delta=\delta(\varepsilon)>0$ such that it satisfies

$$
\frac{1+\varepsilon}{-\ln (1-\mu(p))}>\frac{1}{-\ln (1-(1-\delta) \mu(p))}+\delta,
$$

and assume that $K=K(\delta)$ from Lemma 2.2 satisfies $K>100$, so we can also use Lemma 2.1. Assuming $p=p(n)$ satisfies $n p \rightarrow \infty$ (or even the weaker condition $p>\frac{K^{*}}{n}$ for $K^{*}=e^{K / \delta}$ ), we give a deterministic algorithm, Algorithm CFC (following page), which a.a.s. constructs a conflict-free colouring of $G(n, p)$ using

$$
(1+\varepsilon) \frac{\ln (n p)}{-\ln (1-\mu)}
$$




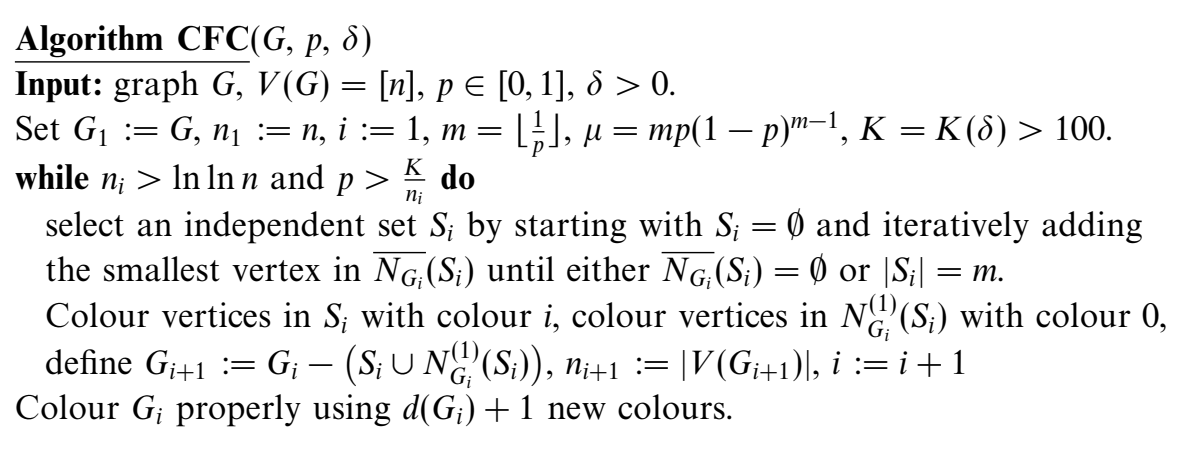

colours. In this algorithm $d\left(G_{i}\right)$ denotes the degeneracy of the graph $G_{i}$, i.e., the largest minimum degree of any non-empty subgraph of $G_{i}$.

Note that all executions of the main while loop of the algorithm use a separate colour and only colour 0 is used in many executions. Note also that this colour 0 is a 'filler colour' as it is never used as the unique colour in the closed neighbourhood of some vertex to ensure the conflict-free property of the colouring is obtained.

Let $I$ be the last value of the index $i$ in the algorithm. Clearly the algorithm colours all vertices with $I+d\left(G_{I}\right)+1$ colours. To see that this colouring is conflict-free let $w \in V(G)$ be an arbitrary vertex and let $i$ be the largest index with $w \in V\left(G_{i}\right)$. If $i<I$, then there is a unique vertex in $N[w]$ of colour $i$ (which may or may not be $w$ itself). If $i=I$, then $w$ has a unique colour in $N[w]$.

To finish the proof it is enough to bound the values of $I$ and $d\left(G_{I}\right)$ a.a.s. We start with $I$. Note that for any $1 \leqslant i \leqslant I$ the sets $S_{1}, \ldots, S_{i-1}$ selected by the algorithm, and hence the vertex set $V\left(G_{i}\right)$ as well, depend only on the edges incident to $S_{1} \cup \cdots \cup S_{i-1}$. Thus, for any way the main while loop is executed for the first $i-1$ times, the graph $G_{i}$ is still a random graph $G\left(n_{i}, p\right)$. Now we estimate the probability that $\left|N_{G_{i}}^{(1)}\left(S_{i}\right)\right|<(1-\delta) \mu\left(n_{i}-m\right)$. This can happen either with $\left|S_{i}\right|=m$ or with $\left|S_{i}\right|<m$. The probability of the former is bounded by Lemma 2.2, while the latter implies that $S_{i}$ is dominating in $G_{i}$, the probability of which is bounded by Lemma 2.1. Using the explicit bounds in the lemmas and the fact that the sizes of the graphs considered are decreasing and lower-bounded by a super-constant function of $n$, we conclude that a.a.s. in no iteration do we have either of these anomalies:

$$
\sum_{i=1}^{I-1} e^{-\delta^{2} \mu n_{i} / 4}+0.9^{n_{i}} \leqslant \sum_{\ell=\ln \ln n}^{n} e^{-\delta^{2} \mu \ell / 4}+0.9^{\ell}=o(1) .
$$

Thus a.a.s. we must have $n_{i+1} \leqslant(1-(1-\delta) \mu) n_{i}$ for each $i<I$. Using $n_{1}=n$ and $n_{I-1}>$ $K / p$, we have a.a.s.

$$
I<\frac{\ln (n p / K)}{-\ln (1-(1-\delta) \mu)}+2
$$

It remains to show that a.a.s. $d\left(G_{I}\right)<\delta \ln (n p)$, and using the defining inequality (2.1) for $\delta$ the upper bound in Theorem 1.1 follows. We use again the observation that, independent 
of the executions of the while loop, $G_{I}$ is a random graph $G\left(n_{I}, p\right)$, with $n_{I}$ being small enough to trigger one of the halting conditions.

If we have $p \leqslant K / n_{I}$, then the expected degree of any vertex in $G\left(n_{I}, p\right)$ is less than $K$. Hence either a.a.s. $d\left(G_{I}\right) \leqslant K$ by the results of Pittel, Spencer and Wormald [8] and Łuczak [6], and we are done, as $K<\delta \ln (n p)$, or $n_{I}$ is bounded by a constant, in which case we can colour $G_{I}$ with $n_{I} \leqslant \delta \ln (n p)$ colours. If, however, $p>K / n_{I}$, we must have $n_{I} \leqslant \ln \ln n$ to halt the while loop, so we have $\ln (n p)>\ln \left(K n / n_{I}\right)=\Omega(\ln n)$. Thus we have $d\left(G_{I}\right)<n_{I}<\delta \ln (n p)$ if $n$ is large enough.

Note that the $\ln \ln n$ bound in the halting condition of the algorithm can be replaced by any function that tends to infinity and is $o(\ln n)$.

Furthermore, observe that if $d\left(G_{I}\right)<\delta \ln (n p)$ (which happens a.a.s.), one can efficiently colour $G_{I}$ with $\delta \ln (n p)$ colours properly. A linear time algorithm for colouring $G_{I}$ with at most $d\left(G_{I}\right)+1$ colours first iteratively removes a lowest degree vertex from the graph, then colours them greedily in reverse order. Hence, the running time of the algorithm CFC is linear in the size of the input graph.

\subsection{Lower bounds}

Tardos and Pach [7] used the concept of universality to show the lower bound $\chi_{C F}\left(G\left(n, \frac{1}{2}\right)\right)=\Omega(\ln n)$. A graph $G$ is called $k$-universal if, for all sets $B \subseteq A \subseteq V(G)$ with $|A| \leqslant k$, there exists a vertex $x \in V(G) \backslash A$ with $N(x) \cap A=B$. We introduce a similar concept that is more closely related to the idea of conflict-free colouring. We call a graph $G(k, f)$-spoiling if, for any $k$ disjoint subsets $A_{1}, \ldots, A_{k} \subseteq V(G)$ with $\left|A_{i}\right| \leqslant f$ for every $i \in[k]$, there exists a vertex $x \in V(G) \backslash \bigcup_{i} A_{i}$ such that for each $A_{i}$ we have $\left|N(x) \cap A_{i}\right| \neq 1$, and for each $A_{i}$ with $\left|A_{i}\right|=f,\left|N(x) \cap A_{i}\right| \geqslant 2$. The vertex $x$ is called an $f$-spoiler for $\left(A_{1}, \ldots, A_{k}\right)$ and we say that $\left(A_{1}, \ldots, A_{k}\right)$ is spoiled by $x$. We call a graph $k$-spoiling if it is $(k, f)$-spoiling for some $f$.

The following observation serves only to provide intuition for the concept.

Observation 2.3. A 2k-universal graph $G$ is $(k, 2)$-spoiling and consequently $k$-spoiling.

The next lemma is the essence of all lower bounds in Theorem 1.1.

Lemma 2.4. If $G$ is $k$-spoiling, then $\chi_{C F}(G)>k$.

Proof. Let $G$ be $(k, f)$-spoiling for some $f$ and consider an arbitrary $k$-colouring $\chi$ of $V(G)$. We need to show that it is not conflict-free. We define subsets $A_{1}, \ldots, A_{k} \subseteq V(G)$. For each colour $i$ which is used less than $f$ times by $\chi$, we define $A_{i}$ to be the whole colour class $\chi^{-1}(\{i\})$. For each colour $i$ which is used on at least $f$ vertices by $\chi$, we set an arbitrary $f$-subset of vertices with colour $i$ to be $A_{i}$. Since $G$ is $(k, f)$-spoiling we find a vertex $x$ which is an $f$-spoiler for these sets. Clearly, $N[x]$ has no unique colour, showing that $\chi$ is not conflict-free.

We first prove the tight lower bound of Theorem 1.2 by studying the spoilers of $G(n, p)$ for $p \geqslant \frac{1}{2}$. Comparing the bound of Theorem 1.3 of Wieland and Godbole [11] and 
Glebov, Liebenau and Szabó [5] with the bound in the following lemma finishes the proof.

Lemma 2.5. The graph $G(n, p)$ with $1 / 2 \leqslant p<1$ is a.a.s. $k$-spoiling for

$$
k=\left\lfloor\frac{\ln n-2 \ln \ln n+\ln \ln \frac{1}{1-p}-\ln 3}{-\ln (1-p)}\right\rfloor .
$$

Proof. We show that $G(n, p)$ is a.a.s. ( $k, 3)$-spoiling. Take any set $A \subseteq V$ with $|A| \leqslant 3$ and $x \in V \backslash A$. For $A=\emptyset$ we cannot have $|N(x) \cap A|=1$. For $|A|=1$ we have

$$
\mathbb{P}[|N(x) \cap A| \neq 1]=1-p,
$$

for $|A|=2$ we have

$$
\mathbb{P}[|N(x) \cap A| \neq 1]=1-2 p(1-p) \geqslant 1-p,
$$

and finally for $|A|=3$ we have

$$
\mathbb{P}[|N(x) \cap A| \geqslant 2]=1-3 p(1-p)^{2}-(1-p)^{3} \geqslant 1-p .
$$

Then for any family $\mathcal{A}=\left\{A_{1}, \ldots, A_{k}\right\}$ of $k$ sets of size at most $f=3$, the probability that a fixed vertex $x \in V \backslash \bigcup_{i=1}^{k} A_{i}$ is a spoiler is

$$
\mathbb{P}[x \text { is a spoiler for } \mathcal{A}] \geqslant(1-p)^{k} \text {. }
$$

Thus

$$
\mathbb{P}\left[\mathcal{A} \text { is not spoiled by any } x \in V \backslash \bigcup_{i=1}^{k} A_{i}\right] \leqslant\left(1-(1-p)^{k}\right)^{n-3 k} .
$$

There are at most $(n+1)^{3 k}$ ways $\mathcal{A}$ can be selected, so by the union bound we have

$\mathbb{P}[G(n, p)$ is not $(k, 3)$-spoiling $] \leqslant(n+1)^{3 k} \exp \left(-(n-3 k)(1-p)^{k}\right)$

$$
\leqslant \exp \left(3 \frac{\ln n-2 \ln \ln n+\ln \ln \frac{1}{1-p}-\ln 3}{-\ln (1-p)} \ln n-n \frac{3 \ln ^{2} n}{-n \ln (1-p)}+o(1)\right)=o(1),
$$

assuming $p \leqslant 1-\frac{1}{n}$. Otherwise $k=0$ and the statement of the lemma becomes trivial, since every graph is 0 -spoiling.

The next lemma provides the lower bound in Theorem 1.1 when $p \leqslant \frac{1}{2}$.

Lemma 2.6. For every $\varepsilon>0$ there exists a constant $K=K(\varepsilon)$ such that, for all $p$ with $K / n \leqslant p \leqslant 1 / 2$, the graph $G(n, p)$ is a.a.s. $k$-spoiling for

$$
k=\left\lfloor(1-\varepsilon) \frac{\ln (n p)}{-\ln (1-\mu)}\right\rfloor \text {. }
$$

Proof. Similarly to the last section, we fix $m=\left\lfloor\frac{1}{p}\right\rfloor$. We show that $G(n, p)$ is a.a.s. $(k, 6 m)$ spoiling. First we observe that, for any fixed $S \subset V$ of size at most $6 m$ and a fixed vertex 
$x \in V \backslash S$, the probability that $x$ spoils $S$ is at least $1-\mu$. Note that $1-\mu$ is exactly the probability if $|S|=m$, and by the definition of $\mu$ as a maximum it is at least this much for other sizes strictly below $6 \mathrm{~m}$. A simple way to see the bound for $|S|=6 \mathrm{~m}$ is to partition $S$ into six parts of size $m$ each. The probability that $x$ has exactly one neighbour in any one of them is $\mu$; these events are independent, so the probability that this holds for at least two of them is exactly $1-(1-\mu)^{6}-6 \mu(1-\mu)^{5}$. Since we have $\mu>e^{-1}$ this is larger than $1-\mu$.

Note that $k<3 \ln (n p)$ since $\mu>1 / e$. First we fix a family $\mathcal{A}$ of $k$ disjoint sets of size at most $6 m$ each and estimate the probability that no vertex $x \in V \backslash \cup \mathcal{A}$ is a spoiler for it:

$$
\begin{aligned}
\mathbb{P}[\nexists x \in V \backslash \bigcup \mathcal{A}: x \text { spoils } \mathcal{A}] & \leqslant\left(1-(1-\mu)^{k}\right)^{n-|\bigcup \mathcal{A}|} \\
& \leqslant \exp \left(-\frac{n}{2}(1-\mu)^{k}\right) \\
& \leqslant \exp \left(-\frac{(n p)^{\varepsilon}}{2 p}\right),
\end{aligned}
$$

where in the second inequality we use the fact that $|\bigcup \mathcal{A}| \leqslant 6 m k<n / 2$ for $K$ large enough.

The union bound for the probability that this happens for any family $\mathcal{A}$ of $k$ sets of size at most $6 \mathrm{~m}$ each is now enough to finish the proof:

$$
\begin{aligned}
\mathbb{P}[\exists \mathcal{A} \forall x \in V \backslash \bigcup \mathcal{A}: x \text { does not spoil } \mathcal{A}] & <\left(\sum_{i \leqslant 6 m}\left(\begin{array}{c}
n \\
i
\end{array}\right)\right)^{k} \exp \left(-\frac{(n p)^{\varepsilon}}{2 p}\right) \\
& <(n p)^{6 m k} \exp \left(-\frac{(n p)^{\varepsilon}}{2 p}\right) \\
& <\exp \left(\frac{18 \ln ^{2}(n p)}{p}-\frac{(n p)^{\varepsilon}}{2 p}\right) \\
& =o(1) .
\end{aligned}
$$

\section{Graphs with large conflict-free chromatic number}

In this section we show the existence of $n$-vertex graphs $G$ with $\chi_{C F}(G)=\Omega\left(\ln ^{2} n\right)$. This gives the correct order of magnitude of $\chi_{C F}(n)$ and proves Theorem 1.4.

To show the statement, we construct an $n$-vertex graph $G$ using random methods. The vertex set is partitioned into classes $L_{1}, \ldots, L_{k}$ of size $\frac{n}{k}$ each, with $k=\lfloor\ln n\rfloor$. The edges will be selected at random, independently of each other. To define the probabilities we let the weight of a vertex $x \in L_{i}$ be

$$
w_{x}=0.99^{i}
$$

The probability of an edge between vertices $x \in L_{i}$ and $y \in L_{j}$ is equal to

$$
\mathbb{P}[x y \in E(G)]:=w_{x} w_{y}=0.99^{i+j} .
$$


The weight of a set $S \subseteq V$ is defined to be the sum of the weights of its elements,

$$
w(S)=\sum_{v \in S} w_{v} .
$$

For a vertex colouring $\chi$ we say that vertex $v$ takes care of itself if the colour of $v$ is unique in $N[v]$, i.e., every $u \in N(v)$ has a colour different from $\chi(v)$. We say that a colour class $S$ takes care of a vertex $x$ if $x \in N^{(1)}(S)$. The crucial probability, denoted by $p(x, S)$, that a vertex $x \in L_{i}$ is taken care of by a colour class $S$ not containing $x$ is equal to

$$
\begin{aligned}
p(x, S)=\mathbb{P}[|N(x) \cap S|=1] & =\sum_{s \in S} \mathbb{P}[N(x) \cap S=\{s\}] \\
& =\sum_{s \in S} w_{s} w_{x} \prod_{y \in S \backslash\{s\}}\left(1-w_{y} w_{x}\right) \\
& <w_{x} \sum_{s \in S} w_{s} \exp \left(-\sum_{y \in S \backslash\{s\}} w_{y} w_{x}\right) \\
& =w_{x} \sum_{s \in S} w_{s} \exp \left(-w(S) w_{x}+w_{s} w_{x}\right) \\
& \leqslant w_{x} w(S) e^{-w_{x} w(S)+0.99} .
\end{aligned}
$$

Note that since the function $z e^{-z}$ has a unique maximum at $z=1$, we always have $p(x, S)<e^{-0.01}$. If $\chi$ is a conflict-free colouring, then every vertex is taken care of either by itself or by a colour class not containing this vertex.

We call a set heavy if its weight is larger than $\sqrt{n}$, otherwise we call it light. Note that since any vertex has weight at least $0.99^{\ln n}>n^{-0.02}$, we obtain for any light colour class $S$

$$
|S|<w(S) n^{0.02}<n^{0.52} .
$$

In the following lemma we list three properties, which hold a.a.s. for our random $G$ and, together, imply that no conflict-free colouring exists with $o\left(\ln ^{2} n\right)$ colours. As usual, $\alpha(G)$ denotes the independence number of $G$, i.e., the size of a largest independent set.

Lemma 3.1. For $G$ the following three properties hold a.a.s.

(i) $\alpha(G) \leqslant n^{0.6}$.

(ii) For every heavy set $S \subseteq V$, we have $\left|N^{(1)}(S)\right|<n^{0.6}$.

(iii) Let $r=\left\lfloor 10^{-5} \ln ^{2} n\right\rfloor$. For all pairwise disjoint light sets $S_{1}, \ldots, S_{r} \subseteq V$, we have

$$
\left|\bigcup_{i=1}^{r} N^{(1)}\left(S_{i}\right)\right|<n-n^{0.7} \text {. }
$$

Proof. (i) Since the probability of each pair of vertices being an edge of $G$ is at least $0.99^{2 \ln n}$, the largest independent set is at most as large as it is in $G\left(n, 0.99^{2 \ln n}\right)$. It is well known (see, e.g., Theorem 11.25(ii) in Bollobás's book [3] for more details) that for $2.27 / n \leqslant p \leqslant 1 / 2$, a.a.s. the largest independent set in $G(n, p)$ has size at most $2 \frac{\ln (n p)}{p}$. Thus, 
the largest independent set in $G$ a.a.s. has size at most

$$
2 \frac{\ln \left(0,99^{2 \ln n} n\right)}{0,99^{2 \ln n}}<n^{0.6}
$$

(ii) Fix a subset $S \subseteq V$ with weight at least $n^{0.5}$ and a set $A \subseteq V \backslash S$ with at least $n^{0.6}$ elements. We estimate the probability that all elements $x \in A$ are in the one-neighbourhood of $S$ :

$$
\begin{aligned}
\mathbb{P}\left[N^{(1)}(S) \supseteq A\right] & =\prod_{x \in A} p(x, S) \\
& \leqslant \prod_{x \in A} w_{x} w(S) e^{-w_{x} w(S)+1} \\
& <\left(n^{0.48} \exp \left(-n^{0.48}+1\right)\right)^{n^{0.6}} \\
& =\exp \left(-n^{1.08}(1+o(1))\right) .
\end{aligned}
$$

(Here we used that $w_{x} w(S)>0.99^{\ln n} n^{0.5}>n^{0.48}$ and that $z e^{-z}$ is decreasing in the interval $[1, \infty)$.) Summing up over all the at most $2^{n} \cdot 2^{n}$ choices of $S$ and $A$, we obtain that the probability that (ii) fails tends to 0 .

(iii) Fix subsets $S_{1}, \ldots, S_{r}$ with $w\left(S_{i}\right) \leqslant \sqrt{n}$ and $B$ with $|B|=n^{0.7}$. We estimate the probability that all $x \in V \backslash B$ are in the one-neighbourhood of at least one of the $S_{i}$.

For this we first show that $\sum_{i=1}^{r} p\left(x, S_{i}\right)>0.01 \ln n$ for at most half of the vertices $x \in V$. Indeed, otherwise

$$
\begin{aligned}
\frac{n}{2} \cdot 0.01 \ln n & \leqslant \sum_{x \in V} \sum_{i=1}^{r} p\left(x, S_{i}\right) \\
& =\sum_{i=1}^{r} \sum_{x \in V} p\left(x, S_{i}\right) \\
& \leqslant r(100 e+100+200) \frac{n}{\ln n}
\end{aligned}
$$

contradicting the definition of $r$. For the last estimate we used that for a fixed colour class $S_{i}$

$$
\sum_{x \in V} p\left(x, S_{i}\right) \leqslant \frac{n}{\ln n} \sum_{j=1}^{\infty} z_{j} e^{-z_{j}+1}
$$

where $z_{j}$ is a geometric progression with quotient 0.99 . The terms of the sum for $z_{j} \leqslant 1$ can be estimated by $e z_{j}$ and hence this part is at most $\frac{e}{1-0.99}=100 e$. The sum of the terms for $z_{j} \geqslant 2$ can be estimated by $100 \int_{1}^{\infty} z e^{-z+1} d z=200$. And finally the sum of the terms for $1<z_{j}<2$ can be estimated by 100 , since there are at most 100 such $z_{j}$, and for each of them the value of the function is at most 1 . 
Let $V^{\prime} \subseteq V$ be the set of those vertices $x \in V$ for which $\sum_{i=1}^{r} p\left(x, S_{i}\right) \leqslant 0.01 \ln n$. Then by the above, $\left|V^{\prime}\right| \geqslant n / 2$ and

$$
\begin{aligned}
\mathbb{P}\left[\forall x \in V \backslash B \exists i \text { with }\left|N(x) \cap S_{i}\right|=1\right] & =\prod_{x \in V \backslash B}\left(1-\prod_{i=1}^{r}\left(1-p\left(x, S_{i}\right)\right)\right) \\
& \leqslant \exp \left(-\sum_{x \in V^{\prime} \backslash B} \prod_{i=1}^{r}\left(1-p\left(x, S_{i}\right)\right)\right) \\
& \leqslant \exp \left(-\sum_{x \in V^{\prime} \backslash B} e^{-5 \sum_{i=1}^{r} p\left(x, S_{i}\right)}\right) \\
& \leqslant \exp \left(-\left(\frac{n}{2}-n^{0.7}\right) e^{-0.05 \ln n}\right) \\
& \leqslant \exp \left(-n^{0.95}(1 / 2-o(1))\right) .
\end{aligned}
$$

Here we used that in the range of our interest, i.e., for $0<z=p\left(x, S_{i}\right)<e^{-0.01}$, we have $1-z>e^{-5 z}$.

The sets $S_{1}, \ldots S_{r}$ and $B$ with the given properties can be chosen at most

$$
\left(\begin{array}{c}
n \\
n^{0.7}
\end{array}\right)\left((n+1)^{\sqrt{n}}\right)^{r}=e^{O\left(n^{0.7} \ln n\right)}
$$

ways, where we first choose a set $B$ of size $n^{0.7}$ from $V$, and then choose one by one the vertices forming the sets $S_{1}, \ldots, S_{r}$. Hence with probability tending to 1 condition (iii) holds.

Finally, we show how the above properties imply the existence of graphs without a conflict-free colouring with $10^{-5} \ln ^{2} n$ colours.

Proof of Theorem 1.4. Let us take a graph $G$ having properties (i)-(iii) of Lemma 3.1 with a sufficiently large vertex set. Take an arbitrary $r$-colouring $c$ of $G$, where $r=\left\lfloor 10^{-5} \ln ^{2} n\right\rfloor$ as in the lemma. We prove that $c$ is not a conflict-free colouring. We define the following sets:

- the set of all vertices that take care of themselves, that is,

$$
T=\{x \in V: \forall y \in N(x), c(x) \neq c(y)\},
$$

- the set of all vertices that are taken care of by a heavy colour class, that is,

$$
H=\left\{x \in V: \exists z \in N(x):\left|c^{-1}(c(z))\right|>\sqrt{n} \wedge \forall y \in N[x] \backslash\{z\}, c(z) \neq c(y)\right\},
$$

- the set of all vertices that are taken care of by a light colour class, that is,

$$
L=\left\{x \in V: \exists z \in N(x):\left|c^{-1}(c(z))\right| \leqslant \sqrt{n} \wedge \forall y \in N[x] \backslash\{y\}, c(z) \neq c(y)\right\} .
$$

If $c$ is a conflict-free colouring, then $V=T \cup H \cup L$. 
Vertices taking care of themselves. A set of vertices that take care of themselves and have the same colour must form an independent set in $G$. Hence by (i) any colour class can contain at most $n^{0.6}$ vertices that take care of themselves. So $|T| \leqslant r n^{0.6}$.

Vertices taken care of by heavy colour classes. Fix a heavy colour class $S$. By (ii) at most $n^{0.6}$ vertices are taken care of by $S$. Hence $|H| \leqslant r n^{0.6}$.

Vertices taken care of by light colour classes. Let $S_{1}, \ldots, S_{r^{*}}$ be the light colour classes of $\chi$. By (iii) at most $n-n^{0.7}$ vertices are taken care of by the $S_{i}$. Hence $|L| \leqslant n-n^{0.7}$.

Thus, $|T \cup H \cup L|<n=|V|$, and $c$ is not conflict-free. This concludes the proof that $c$ is not a conflict-free colouring.

\section{Remarks and open problems}

Radio networks. Recently, Noga Alon pointed out to us that the lower bound from Theorem 1.4 is similar to the one obtained by Alon, Bar-Noy, Linial and Peleg [1]. Here we discuss briefly the relation between the two results. The notation in [1] is different from the one we use here, since they look at a much more applied problem. Creating a small dictionary between the two notations, they speak about processors when we have vertices, a radio network is what we call a graph, transmitting at step $i$ corresponds to having colour $i$, and the transmission itself is a colour class. The problem they analyse is as follows. At the beginning, one processor (the sender) has a message $M$, and the process stops when $M$ is delivered to every processor of the network. The communication in the network works as follows. At step $i$, every processor from transmission $T_{i}$ that has already received $M$ sends it to all adjacent processors. A processor receives a message in a given step if precisely one of its neighbours transmits in this step. If none of its neighbours transmits, it hears nothing. If more than one neighbour transmits, a collision occurs and the processor hears only noise. A sequence of transmissions is a broadcast schedule for the sender $s$ in a network if, after applying the transmissions, every processor in the network has a copy of the message. Alon, Bar-Noy, Linial and Peleg [1] showed that the shortest length of a broadcast schedule is $\Omega\left(\ln ^{2} n\right)$ for some radio networks with $n$ processors. The matching upper bound of $O\left(\ln ^{2} n\right)$ for any radio networks with $n$ processors was established earlier by Bar-Yehuda, Goldreich and Itai [2].

On the one hand, Alon, Bar-Noy, Linial and Peleg [1] do not restrict a processor to be part of only one transmission, while in our setting, a vertex has exactly one colour. On the other hand, in our setting we do not have any scheduling structure, and a vertex does not have to wait until it receives the message to 'transmit'. Hence, none of the lower bounds implies the other immediately. However, observe that in the proof of Theorem 1.4 we do not use the fact that colour classes are disjoint. Consequently our construction gives a common generalization of Theorem 2.1 of [1] and our Theorem 1.4, as follows.

Let us denote by $\chi_{C F}^{\prime}(n)$ the smallest integer such that every graph $G$ on $n$ vertices satisfies the following. There exists a family $\mathcal{F} \subseteq 2^{V(G)}$ of subsets of $V(G)$ of size $|\mathcal{F}| \leqslant \chi_{C F}^{\prime}(n)$ such that, for every vertex $x \in V(G)$, there exists a set $F \in \mathcal{F}$ with $\left|N_{G}(x) \cap F\right|=1$. Both our paper and [1] deal with problems with further requirements on the family $\mathcal{F}$. We insist that they form a partition of the vertex set, whereas Alon, Bar-Noy, Linial and 
Peleg require an ordering of the sets in $\mathcal{F}$ with certain properties to exist. Proving a lower bound for $\chi_{C F}^{\prime}$ therefore implies the corresponding lower bounds in both papers. The construction in [1] has chromatic number 2, so it does not provide a meaningful lower bound for $\chi_{C F}^{\prime} \leqslant \chi_{C F} \leqslant \chi(G)$. The proof of Theorem 1.4 does work in this more general scenario and shows a lower bound of order $\ln ^{2} n$. The corresponding upper bound follows either from [7] or [2].

Theorem 4.1. $\chi_{C F}^{\prime}(n)=\Theta\left(\ln ^{2} n\right)$.

Open problems. At the two extreme values of $p$, the trivial upper bounds given by the chromatic number and the domination number plus one are tight. For the very sparse range of $p=o(1 / n)$ the random graph $G(n, p)$ is a.a.s. a tree, hence both $\chi(G(n, p))$ and $\chi_{C F}(G(n, p))$ are a.a.s. 2 . On the other hand, for $p \geqslant \frac{1}{2}$ we showed that $\left|\chi_{C F}-D\right| \leqslant 3$. The particular questions which remain to be answered are as follows.

- In what range is $\chi_{C F}(G(n, p))=D(G(n, p))+1$ a.a.s.?

- In what range is $\chi(G(n, p))=\chi_{C F}(G(n, p))$ a.a.s.? In particular, we would be interested in where the threshold of 3-conflict-free colourability is and how much it differs, if at all, from the threshold of 3-colourability.

- Does $\chi_{C F}(G(n, p))$ behave in a unimodal way? For example, one might consider the median function and ask whether it is unimodal.

It is an interesting general question to characterize those graphs where equality holds for $\chi_{C F}(G)=\chi(G)$ or $\chi_{C F}(G)=D(G)+1$.

By the concentration results of $[11,5]$ we have the concentration of $\chi_{C F}(G(n, p))$ on two values a.a.s. whenever $\chi_{C F}(G(n, p))=D(G(n, p))+1$. For what range of $p$ does the twovalue concentration hold a.a.s.? We have a concentration on three values a.a.s. whenever $\ln 3 / \ln (1-p) \approx 0$. In the worst case, when $p=1 / 2$, we have concentration on five values a.a.s. For $p \geqslant \frac{\sqrt{5}-1}{2}$, we have concentration on four values a.a.s. (For this we need to consider the $(k, 2)$-spoiling property and adapt the proof of Lemma 2.5.) It would be interesting to obtain a concentration on two values for a wider range of $p$.

\section{Acknowledgement}

We would like to thank Yury Person for pointing out an error in an earlier version of the proof of Theorem 1.4 and Fabian Rabe for useful comments. Furthermore, we are grateful to Noga Alon for drawing our attention to the result on radio networks [1], as well as for fruitful related discussions.

\section{References}

[1] Alon, N., Bar-Noy, A., Linial, N. and Peleg, D. (1991) A lower bound for radio broadcast. J. Comput. System Sci. 43 290-298.

[2] Bar-Yehuda, R., Goldreich, O. and Itai, A. (1986) On the time-complexity of broadcast in radio networks: An exponential gap between determinism and randomization. In Proc. 4th ACM Symposium on Principles of Distributed Computing, pp. 98-107. 
[3] Bollobás, B. (2001) Random Graphs, second edition, Academic Press.

[4] Even, G., Lotker, Z., Ron, D. and Smorodinsky, S. (2003) Conflict-free colorings of simple geometric regions with applications to frequency assignment in cellular networks. SIAM J. Comput. 33 94-136.

[5] Glebov, R., Liebenau, A. and Szabó, T. Concentration of the domination number of the random graph. Submitted. http://arxiv.org/pdf/1209.3115v3.pdf

[6] Łuczak, T. (1991) Size and connectivity of the k-core of a random graph. Discrete Math. 91 61-68.

[7] Pach, J. and Tardos, G. (2009) Conflict-free colorings of graphs and hypergraphs. Combin. Probab. Comput. 18 819-834.

[8] Pittel, B., Spencer, J. and Wormald, N. C. (1996) Sudden emergence of a giant $k$-core in a random graph. J. Combin. Theory Ser. B 67 111-151.

[9] Smorodinsky, S. (2003) Combinatorial problems in computational geometry. PhD Dissertation, School of Computer Science, Tel Aviv University.

[10] Smorodinsky, S. Conflict-free coloring and its applications. In Geometry: Intuitive, Discrete, and Convex (I. Bárány, K. J. Böröczky, G. Fejes Tóth and J. Pach, eds), Vol. 24 of Bolyai Society Mathematical Studies, Springer. To appear. http://arxiv.org/pdf/1005.3616.pdf

[11] Wieland, B. and Godbole, A. P. (2001) On the domination number of a random graph. Electron. J. Combin. 8 R37. 